\title{
Within- and Cross-Modal Integration and Attention in the Autism Spectrum
}

\author{
Geneviève Charbonneau ${ }^{1} \cdot$ Armando Bertone $^{2,3} \cdot$ Marie Véronneau $^{1,7} \cdot$ Simon Girard $^{1} \cdot$ Maxime Pelland $^{1,8}$. \\ Laurent Mottron ${ }^{4} \cdot$ Franco Lepore $^{1} \cdot$ Olivier Collignon ${ }^{5,6}$
}

๑) Springer Science+Business Media, LLC, part of Springer Nature 2019

\begin{abstract}
Although impairment in sensory integration is suggested in the autism spectrum (AS), empirical evidences remain equivocal. We assessed the integration of low-level visual and tactile information within and across modalities in AS and typically developing (TD) individuals. TD individuals demonstrated increased redundancy gain for cross-modal relative to double tactile or visual stimulation, while AS individuals showed similar redundancy gain between cross-modal and double tactile conditions. We further observed that violation of the race model inequality for cross-modal conditions was observed over a wider proportion of the reaction times distribution in TD than AS individuals. Importantly, the reduced cross-modal integration in AS individuals was not related to atypical attentional shift between modalities. We conclude that AS individuals displays selective decrease of cross-modal integration of low-level information.
\end{abstract}

Keywords Autism spectrum $\cdot$ Sensory integration $\cdot$ Visual $\cdot$ Tactile

The autism spectrum (AS) is a complex neurodevelopmental condition that is defined by socio-communicative difficulties and the presence of restricted interests and repetitive behaviours. Although atypical sensory abilities have previously been associated with the AS's behavioural and cognitive profiles, they only became recently considered as one of AS's core clinical features, defined by the hyper or hyporeactivity to different sensory inputs or unusual interests in

Electronic supplementary material The online version of this article (https://doi.org/10.1007/s10803-019-04221-8) contains supplementary material, which is available to authorized users.

Olivier Collignon

olivier.collignon@uclouvain.be

1 Department of Psychology, Centre de Recherche en Neuropsychologie et Cognition (CERNEC), University of Montreal, Montreal, QC, Canada

2 Department of Education and Counselling Psychology, School/Applied Child Psychology, McGill University, Montreal, QC, Canada

3 Perceptual Neuroscience Lab for Autism and Development (PNLab), Montreal, QC, Canada

4 Centre d'Excellence en Troubles Envahissants du Développement de l'Université de Montréal (CETEDUM), Rivières-des-Prairies Hospital, Montreal, QC, Canada sensory aspects of the environment (DSM-5-American Psychiatric Association 2013). Although most research regarding sensory abilities in the AS has focused on unimodal visual or auditory processes, understanding the different aspects of sensory integration and how they relate to characteristic behaviors in the AS is arguably more representative compared to subjective reports in this population (Baum et al. 2015; Stevenson et al. 2016; Wallace and Stevenson 2014; Iarocci and McDonald 2006; Marco et al. 2011). Indeed, individuals with AS often describe a general impression of "sensory overload" and difficulties in processing information

5 Institute of Psychology (IPSY) and Institute of Neuroscience (IoNS), University of Louvain, Louvain-La-Neuve, Belgium

6 Center for Mind/Brain Sciences (CIMeC), University of Trento, Mattarello, Italy

7 Present Address: Université du Québec à Montréal, Montréal, Canada

8 Present Address: Department of psychology of the Acadia University, Nova Scotia, Canada 
when stimuli are received through more than one sensory modality at a time, as is the case when walking in a noisy crowd or being in an environment where several objects stimultaneously stimulate the eyes and ears (O'Neill and Jones 1997; Bonneh et al. 2008). There is growing evidence that cross-modal integration is associated with higher-level socio-communicative functioning, repetitive behavior, and restricted interests in the AS (Baum et al. 2015; Cascio et al. 2016; Stevenson et al. 2017). Atypical sensory integration could arguably have a cascading effect on the subsequent and increasingly complex stages of information processing, ultimately impacting large-scale neural functions mediating communication and social interactions in the AS (Stevenson et al. 2014a; Bahrick 2010; Bahrick and Todd 2011; Noel et al. 2018).

The behavioral outcome of sensory integration is often investigated through simple reaction time (SRT) paradigms, with significant decrease in reaction time (RT) when two or more stimuli are presented simultaneously rather than individually (Todd 1912). This effect is classically referred to as the Redundant Signal Effect (RSE; Hershenson 1962; Raab 1962). Different explanations have been put forward to account for the observation of the RSE. The most common are the Race and the Coactivation models. The Race model proposes that each individual stimulus elicits an independent detection process. For a given trial, the fastest stimulus determines the observable RT. On average, the time to detect the fastest of several redundant signals is faster than the detection time for a single signal. Therefore, the speeding up of reaction time is attributable to statistical facilitation. When the prediction of the Race model is violated and the activation of two stimuli in combination induces faster responses than simple probabilistic summation, the speed-up in reaction times is associated with some form of sensory integration [although whether it represents the merging of the signals into a single signal before the decision, or whether it relates to specific neurophysiological processes, remains debated (Otto and Mamassian 2012)].

Although several studies have assessed cross-modal integration in AS individuals (Collignon et al. 2013), our understanding of atypical integration in this population remains equivocal (Wallace and Stevenson 2014). One hypothesis proposes that the ability of AS individuals to integrate crossmodal information is contingent on the type of information that is integrated (Magnée et al. 2008; Bebko et al. 2006; Mongillo et al. 2008). Specifically, it is hypothesized that less efficient integrative abilities are specific to social information in AS (i.e. emotions, language, voices, etc.), possibly due to the more complex and variable nature of social stimuli (Adolphs 2002; Dawson et al. 2004). In contrast, the two studies that used an RSE paradigm and the Miller's race model analysis previously described have demonstrated a deficit in integration of low-level, non-social multisensory inputs in AS children (Brandwein et al. 2013, 2015). They demonstrated that performance in the multisensory condition exceeded that predicted by the race model in TD children, but not in those with an AS diagnosis. This finding is in favor of a general alteration in cross-modal integration in children on the AS regardless of the social versus non-social nature of the stimuli being processed, although those were not directly compared in the studies.

One limitation of those aforementioned studies that concluded in alteration in cross-modal integration in AS children is that they did not ascertain if this result could by explain by a difference between the groups in the capacity to engage and disengage their attention to separate sensory inputs. In TD individuals, the perception of a stimulus is largely influenced by previously presented sensory inputs (Miller 1982; Otto and Mamassian 2012; Gondan et al. 2004). RTs to a sensory input are faster when preceded by a stimulus from the same modality. In contrast, when the stimuli in successive trials originate from different modalities, attention needs to shift from one modality to another, resulting in longer RTs. The cognitive effort associated to the change in attention between modalities on consecutive trials is referred as the modality shift cost. This may explain why, at a perceptual level, participants respond faster to simultaneously presented bimodal signals compared to unimodal signals (Gondan et al. 2004; Spence et al. 2001; Harrar et al. 2014; Otto and Mamassian 2012). In order to specifically assess cross-modal integration atypicalities in AS individuals, it is critical to evaluate whether it originates from a difference in the way they engage and disengage their attention to separate sensory inputs (Harrar et al. 2014; de Heering et al. 2016). A decrease or absence of the modality shift cost in AS individuals could lengthen the response speed for bimodal stimuli compared to TD individuals and therefore partly explain the alteration in cross-modal integration reported in previous studies. This is particularly crucial since studies have shown that the ability to shift or disengage attention is often impaired in AS individuals (i.e., Courchesne et al. 1994; Landry and Bryson 2004; Renner et al. 2006; Wainwright-Sharp and Bryson 1993; Occelli et al. 2013).

Major cognitive theories in autism research, including the Weak Central Coherence theory (Frith and Happe 1994), the Enhanced Perceptual Functioning theory (Mottron et al. 2006), and the temporal binding deficit hypothesis (Brock et al. 2002), emphasize that locally-oriented sensory processing in AS individuals is associated with superior performance on tasks where local analysis is advantageous, with concurrent inferior performance on tasks requiring an integrative approach (Dakin and Frith 2005; Behrmann et al. 2006). It was suggested that the AS was associated with reductions in global connectivity, with typical or even 
increased connectivity within local networks (Brock et al. 2002). These models support the hypothesis of an alteration of cross-modal integration in individuals with AS (Wallace and Stevenson 2014), since this perceptual process require an integrative approach and is based on the combination of information processed from distinct, and often distant, brain networks. This leads to the important question of whether the putative sensory integration atypicalities observed in AS are specific to cross-modal conditions, or if they also apply to within-modal interactions. Detection RT is faster with multiple compared to single stimulus, whether they are presented in the same or in a different sensory modality (Todd 1912; Raab 1962). Most research in TD individual suggest that within- and cross-modal integration differ by the amount of facilitation they produce at the behavioral and neurophysiological level. One hypothesis is that stimuli originating from different senses provide independent estimates of the same event, while there is significant covariance in the information received from stimuli of the same sensory modality (Ernst and Banks 2002; Girard et al. 2013). It was demonstrated that during perceptual tasks, the RG for crossmodal pairs exceeds that of within-modal pairs (Girard et al. 2013; Forster et al. 2002; Laurienti et al. 2006; Miniussi et al. 1998), and performance for within-modal conditions generally did not surpass that predicted by the race model (Girard et al. 2013; Murray et al. 2001; Corballis 1998; Reuter Lorenz et al. 1995; Badzakova-Traikov et al. 2005), which can be explained by statistical facilitation. This is compatible with neurophysiological studies showing that, unlike cross-modal stimuli, within-modal pairs rarely lead to an improvement in neural response (Alvarado et al. 2007a, b). Differences regarding within-modal and cross-modal integration have yet to be directly compared in AS individuals. Based on the theoretical models previously described, we hypothesize that sensory integration atypicalities observed in the AS will be specific to cross-modal conditions because they involve combining signals coming from multiple and more distant cortical areas, and that within-modal interactions, which are mediated by the same cortical areas, will be intact (see Simon and Wallace 2016, for review).

Somatosensory processing is known to play a crucial role in the social development of TD individuals (Maitre et al. 2017; Myers 1984; Hertenstein 2002; Hertenstein et al. 2006; Thye et al. 2018; Dunbar 2010), notably for the pre-verbal socio-communicative development (Cascio et al. 2016; Field 2001), and may contribute to the acquisition of a sense of self (Schütz-Bosbach et al. 2009) which underlies some social skills, including imitation and empathizing (Schütz-Bosbach et al. 2006). Despite the importance of somatosensory perception on such higher-level abilities, the literature on multisensory processing in the AS has mainly been confined to the auditory and visual modalities, possibly because of their obvious role in verbal and nonverbal communication that is frequently found to be atypical in the AS (Baum et al. 2015; Cascio et al. 2016). However, somatosensory atypicalities are among the most frequently reported sensory complaints in the AS (Rogers et al. 2003; Tomchek and Dunn 2007) and mechanisms mediating lowlevel somatosensory perception in AS individuals have been found to be atypical (i.e., Khan et al. 2015; Puts et al. 2017) and related to AS symptomology (Tavassoli et al. 2016). It is therefore highly relevant to include the tactile modality when investigating cross-modal integration in AS individuals.

In the present study, we used a visuo-tactile RSE paradigm to simultaneously assess and compare the ability of AS and TD individuals to detect and integrate simple visual and tactile stimuli both within and across modalities, and to shift attention from one modality to another during multisensory processing. We used easily detectable, suprathreshold stimuli to measure performance in both groups that were subjected to three different types of analyses: (i) reaction times and redundancy gains, (ii) Miller's race model analyses, and (iii) modality switch cost analyses (see the Experimental Procedures for details). Crucially, the redundancy gain associated with visuo-tactile integration in AS individuals was compared to that associated to the integration of redundant visual and tactile stimuli, allowing us to directly compare within- and cross-modal integration performances in the AS.

In both groups, we anticipate a lower redundancy gain in the within- compared to cross-modal conditions, and that performance will not exceed the predictions of the race model for within-modal stimuli. We however hypothesize that cross-modal integration will be selectively altered in AS individuals, resulting in reduced redundancy gain and reduction in the violation of the race model in the AS group. We predict that this alteration will be specific to cross-modal conditions, and that the ability to integrate multiple information coming from the same modality will be preserved in AS individuals. We will also investigate whether a reduced modality shift cost is present in AS compared to TD individuals, which could partially explain their selective deficit in multisensory integration.

\section{Methods}

\section{Participants}

Fourteen AS individuals and fourteen typically developing (TD) controls participated in this study. Participants were recruited from the database of the Rivière-des-Prairies Hospital's autism clinic (Montréal, Canada). Thirteen participants from the AS group were diagnosed utilizing the Autism Diagnostic Interview (ADI-R; Lord et al. 1994) and/ or the Autistic Diagnostic Observation Schedule (ADOS-G; Lord et al. 2000) (ADI only: two; ADI and ADOS: eleven). 
One participant from the AS group was diagnosed based on DSM-IV-TR criteria (American Psychiatric Association 2000) and expert clinical judgment. Trained clinical professionals working at the specialized clinic at the Rivièredes-Prairies Hospital conducted both diagnostic tests. TD participants and their first-degree relatives were screened with a questionnaire for any history of neurological or psychiatric disorders. All participants in both groups were males and had normal, or corrected to normal, near and far vision assessed before testing using near and far Snellen acuity charts. The groups were matched as closely as possible in terms of age (AS: mean age 25 years \pm 5 ; range $18-35$ years; TD: mean age 24 years \pm 4 ; range $16-30$ ), laterality (AS: 1 left-handed participant; TD: 2 left-handed participants) and global Wechsler intellectual quotient [(Full-scale IQ: AS: mean $100 \pm 13$, range $84-126$; TD: mean $111 \pm 13$, range 87-130); (Performance IQ: AS: mean 104 \pm 13 , range 77-123; TD: mean 109 \pm 12 , range 82-131); (Verbal IQ: AS: mean $99 \pm 16$, range $72-128$; TD: $112 \pm 10$, range 94-125)]. All participants had a full-scale Wechsler score of 80 or more. Each participant gave informed written consent to participate in the study and received monetary compensation for this. The ethics board of the Rivière-des-Prairies Hospital approved the study.

\section{Stimuli}

Tactile stimuli were delivered using a pneumatic stimulator that was located outside the testing room in order to reduce any distraction produced by the system. Rigid plastic tubes connected the stimulator to four plastic membranes (one centimeter of diameter) positioned on the ventral surface of the particpants' distal third of their index and the middle fingers. Tactile stimulation consisted of inflation of the plastic membrane by a pulse of air pressure sent through the plastic tube from the pneumatic stimulator. Each tactile stimulation lasted for $100 \mathrm{~ms}$. Given the significant interindividual variability in the sensitivity to tactile stimulation, the intensity at which it was delivered was calibrated individually, according to participant self-report, to obtain reliable stimulations (see Girard et al. 2013). This procedure resulted in a mean pressure of approximately $14.1 \mathrm{kPa}$ in the AS group (range from 12.1 to 19.5 ) and of $14.9 \mathrm{kPa}$ in the TD group (range from 12.1 to 20.6). No significant between-groups difference in threshold was found $(t(23)=-.71, p=.49)$. Participants' hands were positioned at a distance of $57 \mathrm{~cm}$ from their head. The tips of their index fingers were placed at $3^{\circ}$ of eccentricity below a central fixation cross, and at $2.5^{\circ}$ to the left (index of the left hand) or the the right (index of the right hand) of it. The tips of their middle fingers were placed at $3^{\circ}$ of eccentricity above the central fixation cross, and at $2.5^{\circ}$ to the left (middle finger of the left hand) or to the right (middle finger of the right hand) (Fig. 1). Participants' fingers were positioned in a fixed apparatus $(150 \times 120 \times 75 \mathrm{~mm})$. The index finger and the middle finger of the left hand were inserted in separe holes $(25 \mathrm{~mm}$ of diameter and $35 \mathrm{~mm}$ of depth) located on the left side of the box, with a distance of $60 \mathrm{~mm}$ between the tips of both fingers. The same configuration was used on the right side of the box for the index and the middle finger of the right hand.

Visual stimuli were presented on a plastic board $(120 \times 150 \mathrm{~mm})$ located on the top of the apparatus via a projector suspended on the ceiling of the testing room, approximately $50 \mathrm{~mm}$ above the fingertips of the participants. Each visual stimulation consisted in white circle subtending $1^{\circ}$ of visual angle presented on a black background, and lasted for $100 \mathrm{~ms}$. As with tactile stimulations, visual stimuli were delivered at $3^{\circ}$ degrees of eccentricity below or above the central fixation cross, and at $2.5^{\circ}$ to its left or to its right (Fig. 1). For the misaligned (opposite hemifield) double visual and visuo-tactile conditions (see Procedure below for a description of the experimental conditions), visual stimuli were presented at $1.5^{\circ}$ of eccentricity below or above the central fixation cross, and at $2.5^{\circ}$ to its left or to its right, in order to maintain a similar interstimuli distance for the aligned (same hemifield) and the misaligned (opposite hemifield) conditions.
Fig. 1 a Schematic view of the experimental setup and stimulation conditions. Tactile stimuli were delivered to the index and middle fingers of each hand, and visual stimuli were projected on a surface above the stimulated fingers. b Simplified illustration representing the position of the participant and the apparatus

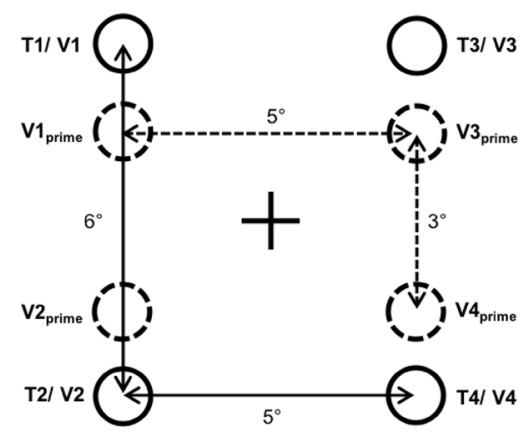

STIMULATION CONDITIONS

Single Modality

Tactile: $\mathrm{T} 1 ; \mathrm{T} 2 ; \mathrm{T} 3 ; \mathrm{T} 4$

Visual: $\mathrm{V} 1 ; \mathrm{V} 2 ; \mathrm{V} 3 ; \mathrm{V} 4$

Within-Modal

Double Visual (Aligned): V1/V2 : V3/V4

Double Visual (Misaligned): $\mathrm{V} 1_{\text {prime }} / \mathrm{V} 4_{\text {prime }} ; \mathrm{V} 2_{\text {prime }} \mathrm{V} 3_{\text {prime }}$

Double Tactile (Aligned): T1/T2; T3/T4

Double Tactile (Misaligned): T1/T4; T2/T3

Cross-Modal

Visuo-Tactile (Aligned): V1/T2 ; V2/T1 ; V3T4 ; V4T3

Visuo-Tactile (Misaligned): $\mathrm{V} 1_{\text {prime }} / \mathrm{T} 4 ; \mathrm{V}_{\text {prime }} / \mathrm{T} 1 ; \mathrm{V} 2_{\text {prime }} / \mathrm{T} 3 ; \mathrm{V} 3_{\text {prime }} / \mathrm{T} 2$ 


\section{Procedure}

The paradigm was adapted from Girard et al. (2013). Participants sat in a silent and darkened room with their head positioned on a chinrest. They were instructed to press on a response button with their left or their right thumb as fast as possible each time a stimulation was presented. The hand used to respond alternated between each block, and the order was counterbalanced across participants. Participants' gaze was monitored throughout the experiment via a camera to ensure that participants maintained central fixation. Stimuli were displayed, and RTs were recorded using Presentation software (Neurobehavioral Systems, Inc., Albany, USA).

Participants were presented with eight different conditions: (1) simple tactile stimulus (T1, T2, T3, T4); (2) aligned double tactile stimuli (T1/T2, T3/T4); (3) misaligned double tactile stimuli (T1/T4, T2/T3); (4) simple visual stimulus (V1, V2, V3, V4); (5) aligned double visual stimuli (V1/V2, V3/V4); (6) misaligned double visual stimuli $\left(\mathrm{V} 1_{\text {prime }} / \mathrm{V} 4_{\text {prime }}, \mathrm{V} 2_{\text {prime }} / \mathrm{V} 3_{\text {prime }}\right)$; (7) aligned visuo-tactile stimuli (V1/T2, V2/T1, V3/T4, V4/T3) and (8) misaligned visuo-tactile stimuli $\left(\mathrm{V} 1_{\text {prime }} / \mathrm{T} 4, \mathrm{~V} 4_{\text {prime }} / \mathrm{T} 1, \mathrm{~V} 2_{\text {prime }} / \mathrm{T} 3\right.$, $\mathrm{V} 3_{\text {prime }} / \mathrm{T} 2$ ). Aligned conditions consisted of two stimuli presented in the same hemispace (left or right), while misaligned conditions were made up of two stimuli from opposite hemispace. For the aligned conditions, one of the stimuli was presented on (tactile) or above (visual) the index finger of one hand, and the other was presented on (tactile) or above (visual) the middle finger of the same hand. For the misaligned conditions, one of the stimuli was presented on (tactile) or above (visual) the index finger of one hand, and the other was presented on (tactile) or above (visual) the middle finger of the opposite hand. Participants completed six blocks of 260 experimental trials. Each block contained 10 repetitions of the 24 stimuli configurations, and 20 catch trials (8\%), during which no stimulation was presented to restrict the tendency of participants to produce anticipatory responses. A total of 60 trials per condition were therefore recorded. Inter-trial interval randomly varied between 1500 and $3500 \mathrm{~ms}$ (mean $2500 \mathrm{~ms}$ ). The central fixation cross was displayed throughout the experiment. Each block lasted approximately $11 \mathrm{~min}$. Breaks were encouraged between the blocks to help maintain an optimal level of attention.

\section{Data Analysis}

Since the effect of alignment was the same for both groups of participants, the scores for aligned and misaligned conditions were combined for all analysis (see Supplementary Material for the detailed statistical analysis and separate graphical representation of aligned and misaligned conditions).

\section{Reaction Times and Accuracy}

Both accuracy and response speed were examined in order to investigate the general ability to detect sensory inputs. Task accuracy was estimated by the proportion of detected stimuli (in percent). Only RTs ranging from 100 to $1000 \mathrm{~ms}$ were analyzed, otherwise they were discarded from the analysis ( 0.24 and $0.21 \%$ of RTs in AS and TD groups, respectively). In experiments that place equal emphasis on accuracy and processing speed, it is possible that particpants adopt different response strategies by varying RTs inversely with accuracy (and thus resulting in a speed/accuracy trade-off). We therefore calculated the Inverse Efficiency (IE) scores which constitute a standard approach to combine mean RTs and accuracy measures of performance (Townsend and Ashby 1978), and considered as "corrected RTs" that discount possible criterion shifts or speed/accuracy trade-offs. The IE scores were obtained by dividing RTs by the proportion of detected stimuli separately for each condition, with higher IE scores indicated worse performance. IE scores were submitted to repeated measures ANOVAs 2 (Group: AS, TD; between-subjects factor) $\times 5$ (Condition: simple tactile, double tactile, simple visual, double visual, visuo-tactile). Based on significant F-values, Bonferonni post hoc analyses were performed when appropriate. For the sake of completeness, a similar ANOVA was also carried out for accuracy and RTs and are presented as supplemental materials.

\section{Within-Modal and Cross-Modal Integration}

Sensory integration was first investigated by calculating the redundancy gain (RG), which corresponded to the decrease (in percent) in the mean IE scores obtained in the redundant conditions as compared to the mean IE scores obtained for the best single condition (Stein and Meredith 1993; Girard et al. 2011; Charbonneau et al. 2013a, b). For each condition and each participant separately, the mean IE score for the redundant condition was substracted from the mean IE score for the fastest stimulus of the pair, and then divided by the latter, which yielded to the percentage of decrease in IE score between the redundant condition and its best constituent $[\mathrm{RG}=(\mathrm{IE}$ score best single condition $-\mathrm{IE}$ score redundant condition)/IE score best single condition]. The RGs were then submitted to a 2 (Group: AS, TD; betweensubjects factor) $\times 3$ (Modality: double tactile, double visual, visuo-tactile) repeated measures ANOVA.

Analyses of violation of the race model inequality were carried out using the RMITest software which implements the algorithm described in Ulrich et al. (2007). The algorithm estimates the cumulative probability distributions of 
RTs in the two unimodal simple conditions and the redundant condition, and tests whether redundant-targets RTs are significantly faster than would be predicted by a race model (with t-tests). We then calculated the proportion of percentiles showing faster RTs than the prediction of the race model, and compared it using a 2 (Group: AS, TD; betweensubjects factor) $\times 3$ (Modality: double tactile, double visual, visuo-tactile) repeated measures ANOVA.

\section{Modality Switch Cost}

Since one of the effects potentially driving violation of the race model is the cost of switching attention between modalities (Otto and Mamassian 2012; Gondan et al. 2004), we compared the modality switch cost in AS and TD groups. To determine whether the RTs to a stimulus were influenced by the modality of the stimulus presented in the previous trial, the RTs obtained in the single and within-modal conditions were classified in function of their previous trial (previous trial of the same modality and previous trial of a different modality). The modality switch cost was then calculated by subtracting the mean RTs for conditions in which the stimuli were preceded by a stimulus of the same modality to the mean RTs for conditions in which the stimuli were preceded by a stimulus of a different modality (Harrar et al. 2014; Otto and Mamassian 2012). These scores were submitted to a 2 (Group: AS, TD; between-subject factor) $\times 2$ (Modality: tactile, visual) repeated measures ANOVA, in order to test whether the modality switch cost differed according to the group or the modality of the stimulation.

We also carried out a supplemental analysis to investigate whether RTs to a specific stimulus were influenced by the location of the stimulus presented in the previous trial and across all modalities in both groups. RTs were submitted to a 2 (Group: AS, TD; between-subject factor) $\times 2$ (alignment of the previous trial: same hemifield, opposite hemifield $) \times 2$ (Modality: tactile, visual) repeated measures ANOVA.

\section{Results}

\section{Reaction Times and Accuracy}

Overall, both groups performed the detection task accurately. TD particpants detected $97.5 \%$ of all tactile stimuli (range from 91 to $100 \%$ ), $98 \%$ of visual stimuli (range from 92 to $100 \%$ ) and $98 \%$ of cross-modal pairs (range from 92 to $100 \%$ ). AS particpants detected $96 \%$ of tactile stimuli (range from 87 to $100 \%$ ), $97 \%$ of visual stimuli (range from 87 to $100 \%$ ) and $98 \%$ of visuo-tactile stimuli (range from 91 to $100 \%)$. TD particpants responded to $4 \%$ of the catch trials throughout the experiment, whereas AS particpants responded to $2 \%$ of the trials $(t(26)=-1.419, p=.17)$ (see Supplementary Material for detailed analysis on accuracy and RTs separately and a table of the breakdown of the mean accuracy, RTs, IEs and SD across groups).

When comparing IE scores across groups and conditions (Fig. 2a), we found main effects for both the "group" $(F(1,26)=4.90, p=.05, n 2$ partial $=.16)$ and "condition" factors $(F(4,104)=39.03, p \leq .001, n 2$ partial $=.60)$. There was an interaction between those two factors $(F(4,104)=3.31, p=.01, n 2$ partial $=.11)$, as differences in performance between the conditions of presentation changed according to the group. Using post hoc t-tests and applying Bonferroni correction, for the TD group, we found lower IE scores for visuo-tactile conditions than for simple tactile $(p \leq .001)$ and simple visual $(p \leq .001)$ conditions whereas in AS group, there was no significant difference in performance between cross-modal, within-modal and simple visual and tactile conditions.

\section{Within-Modal and Cross-Modal Integration}

RGs were significantly different from 0 for the three redundant conditions in both TD (double tactile: $t(13)=7.26, p \leq .001, n 2$ partial $=.66$; double visual: $t(13)=13.16, p \leq .001, n 2$ partial $=.87$; visuo-tactile: $t(13)=13.40, p \leq .001, n 2$ partial $=.87)$ and AS groups (double tactile: $t(13)=7.83, p \leq .001, n 2$ partial $=.69$; double visual: $t(13)=5.42, p \leq .001, n 2$ partial $=.51$; visuo-tactile: $t(13)=7.36, p \leq .001, n 2$ partial $=.67$ ) When comparing RGs between groups and modalities (Fig. 2b), we found no main effect for the "group" factor $(F(1,26)=.03, p=.88), \quad$ but there was a main effect of the factor "modality" $(F(2,52)=30.08, p \leq .001, n 2$ partial $=.54)$

Moreover we observed a significant interaction between the factors modality and group $(F(2,52)=3.11, p=.02, n 2$ partial $=.11)$ showing that cross-modal visuo-tactile stimuli produced greater RG compared to both double tactile $(p=.003)$ and double visual conditions $(p \leq .001)$ in the TD group, while RGs for the visuotactile and the double tactile conditions did not differ in AS $(p=1.0)$ but were both superior than the RG in the double visual condition $(p \leq .001)$.

To further test for group differences in the within and cross-modal integration conditions, we examined whether the RTs obtained in the redundant conditions exceeded the statistical facilitation predicted by Miller's race model inequality (Miller 1982). For the cross-modal visuo-tactile conditions, the race model inequality was significantly violated up to the 50th percentiles of the RTs distribution in TD (Fig. 2c), while it was only significantly violated for the 10th to the 30th percentiles of the RTs distribution in AS (Fig. 2c). Consistent with previous results (Girard et al. 2013), no significant violation of the race model inequality 
(A)

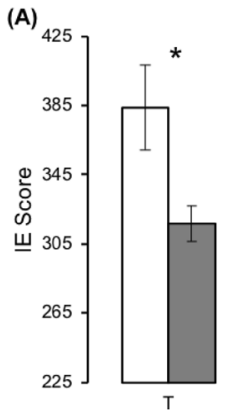

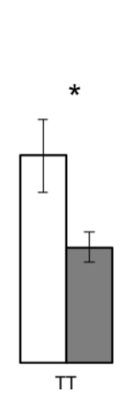

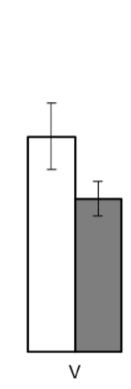

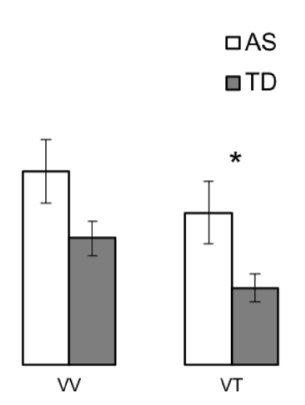

AS

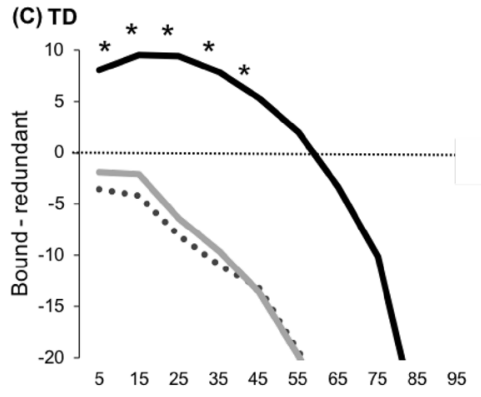

Percentiles of the reaction times distribution
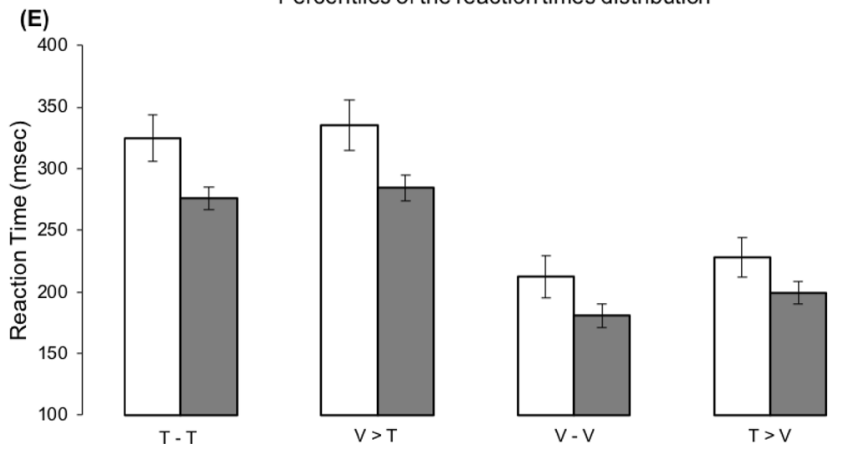

(B)
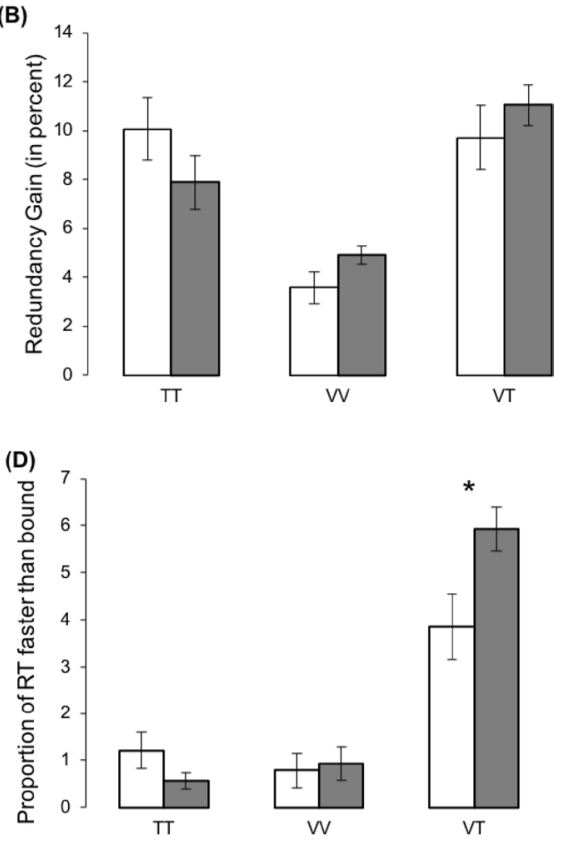

(F)

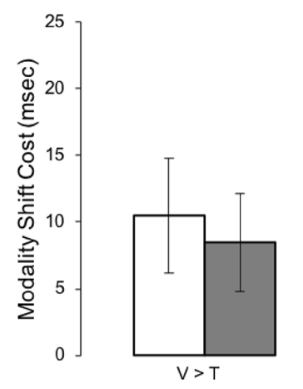

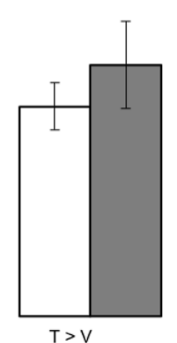

Fig. 2 a Mean IE scores (in $\mathrm{msec}$ ) for single, within-modal and cross-modal conditions for TD and in AS individuals. Error bars represented standard error of the mean. Capital letters referred to the modality ( $T$ tactile, $T T$ double tactile, $V$ visual, $V V$ double visual, $V T$ visuo-tactile). b Mean redundancy gains (RGs) for double within- and cross-modal conditions in TD and AS groups. Error bars represented the standard error of the mean. $\mathbf{c}$ Test for violation of the race model inequality (Miller 1982) in TD (left panel) and in AS (right panel). $\mathrm{Y}$ axis represented the difference in msec between the race model prediction computed from the RT of each single sensory component (or the model bound) and the RT obtained in the redundant conditions for within- and cross-modal pairs. This value was computed for each percentile of the RT distribution represented on the $X$ axis. Positive values on the $\mathrm{Y}$ axis of the graph referred to RTs that were faster than the race model prediction. When this difference was statis-

was demonstrated for the double visual and the double tactile conditions, suggesting that the faster RTs in these conditions could be explained by simple probability summation for both AS and TD participants.

When assessing whether the proportion of percentiles where RTs were faster than predicted by the race model (Fig. 2d), we observed a group by modality interaction $(F(2,52)=8.01, p \leq .001, n 2$ partial $=.24)$, suggesting tically significant, RTs were marked with an asterisk, which indicated a violation of the race model inequality. Negative values on the $\mathrm{Y}$ axis of the graph referred to RTs that were slower than the race model prediction. d Mean proportion of percentiles (out of ten) for which RTs were faster than predicted by the race model for double withinand cross-modal conditions in TD and AS groups. Error bars represented the standard error of the mean. e Modality Shift Cost effects for single and within-modal conditions were calculated according to whether the previous stimulus was from the same or from a different modality, in TD and AS groups. f Modality Shift Cost indexes (in msec) calculated by subtracting the mean RTs for conditions in which the stimuli were preceded by a stimulus of the same modality to the mean RTs for conditions in which the stimuli were preceded by a stimulus of a different modality, as a function of the modality in both groups

that the proportion of percentiles for which the race model was violated was significantly higher in TD individuals compared to AS individuals for the cross-modal visuo-tactile condition. There were no group-difference for the double tactile and double visual conditions. 


\section{Modality Switch Cost}

The modality switch cost for tactile stimuli referred to the mean RTs for conditions in which visual stimuli were preceded by a tactile stimulus when compared to the mean RTs for conditions in which the visual stimuli were preceded by a visual stimulus. The modality switch cost for visual stimuli referred to the mean RTs for conditions in which tactile stimuli were preceded by a visual stimulus when compared to the mean RTs for conditions in which the tactile stimuli were preceded by a tactile stimulus. The modality switch cost was significantly different from 0 for the two modalities in both AS (visual: $t(13)=2.44, p=.03, n 2$ partial $=.18$; tactile: $t(13)=8.81, p \leq .001, n 2$ partial $=.74)$ and TD groups (visual: $t(13)=2.32, p=.04, n 2$ partial $=.17$; tactile: $t(13)=5.77, p \leq .001, n 2$ partial $=.55)$. This confirmed that the RTs are faster when stimuli are preceded by a stimulation of the same, rather than different modality (Fig. 2e, f). We found no main effect for the "group" factor $(F(1,26)=.03, p=.86)$. We observed a main effect of the "modality" factor $(F(1,26)=4.64, p=.04, n 2$ partial $=.15)$, as participants in both groups were faster to switch from visual to tactile stimuli. There was no interaction between these two factors $(F(1,26)=.50, p=.49)$.

We also performed a supplemental analysis to investigate whether RTs to a specific stimulus were influenced by the location of the stimulus presented in the previous trial and across all modalities in both groups. We found no main effect of the spatial alignment $(F(1,26)=.15, p=.70)$ and no interaction between the alignment and the group $(F(1$, $26)=2.18, p=.15)$ or the modality $(F(3,78)=1.16, p=.33)$.

\section{Discussion}

We investigated the ability of AS individuals to integrate cross-modal and within-modal low-level information, and to shift attention from one modality to another in a task requiring the detection of visual and tactile stimuli. Our results demonstrate that AS individuals can integrate cross-modal visuo-tactile information, although this integrative process is decreased compared to TD individuals. When comparing within-modal and cross-modal integration performances across groups, we found that cross-modal visuo-tactile stimuli (or conditions) produced greater RTs facilitation compared to both double tactile and visual conditions in TD individuals, while redundancy gains for visuo-tactile and double tactile were both superior to those in the double visual condition in AS individuals. Importantly, the attentional processes needed to shift from one modality to another were not found to differ between TD and AS groups, suggesting that the decrease in cross-modal integration observed in
AS individuals is not due to an impaired capacity to switch attention across the senses.

\section{Cross-Modal Integration in AS}

The RTs probability for the cross-modal condition exceeded the prediction of the race model for the fastest latencies in both groups, but to a lesser extent in AS compared to TD. Our results therefore demonstrate that AS adults can integrate visuo-tactile information, although this integrative process is less efficient.

We also found that overall, AS participants responded more slowly than controls to any sensory stimuli (see SFig. 1). Nevertheless, application of the race model is advantageous to factor out this unisensory delay since integration is calculated on probability summation of individual unisensory reaction times distributions (see Harrar et al. (2014) for similar discussion in dyslexic individuals). Moreover, according to the inverse effectiveness principle (Stein and Meredith 1993), a basic concept in sensory integration stating that multisensory gain is inversely proportional to saliency of unisensory signals, one may have predicted greater cross-modal integration in AS particpants based on their slower RTs for single visual and tactile conditions, which reinforce our results of a specific decrease of the cross-modal integration process in the AS (Brandwein et al. 2013, 2015; Charbonneau et al. 2013).

Until now, cross-modal integration of visuo-tactile information in AS has mainly been investigated based on visuo-tactile illusions, like the rubber hand illusion (Botvinick 2004), in which information from vision and touch are merged to influence proprioception and body representation. The experience of this illusion has been shown to be reduced in AS individuals (Paton et al. 2012) and delayed in time (Cascio et al. 2012), which may result from reduced cross-modal integration abilities. Results from our study suggest that the decrease in cross-modal integration also extent to the processing of low-level stimuli during a simple detection task. To our knowledge, only the studies of Poole et al. $(2015,2018)$ have used an approach void of socially salient stimuli to investigate visuo-tactile processing in AS individuals. In these studies, participants were asked to judge whether a tactile stimulation was a single or two separate (double) vibrations while presented with a congruent or incongruent number of distracting light flashes, a paradigm somewhat analogous to the sound-induced flash illusion (Shams et al. 2000). Their results demonstrated that AS individuals showed an increased influence of visual distractors on tactile judgments, with a difficulty to suppress the processing of distant contralateral distractors. Interestingly, the influence of distractors of another sensory modality on performance suggest that AS can integrate multisensory 
information, although this process may be atypical, which is also what we observed in this experiment.

The current study is the first to rely on a simple reaction time paradigm in AS adults that allow to measure responses for both within-modal and cross-modal conditions to simple low-level visuo-tactile stimuli, and to directly compare the benefits of multisensory information to a predicted model (i.e. the race model; Miller 1982, 1986) based on responses to the unisensory conditions (see Brandwein et al. $(2013,2015)$ for similar paradigm with audio-visual stimuli in children and adolescents). Some studies based on audio-visual interactions suggested an improvement in the ability to integrate cross-modal sensory information with development in AS (Foxe et al. 2015; Taylor et al. 2010). It is interesting to note that we found reduced violation of the race model inequality for the fastest latencies of the RTs distribution in AS adults, while a complete absence of violation of the race model inequality was found when performing a similar task in AS children and adolescents aged between 7 and 16 years old (Brandwein et al. 2013, 2015). Although speculative, these results may suggest that developmental changes due to neurobiological or experiential factors (or both), progressively regularize cross-modal integration abilities in AS (Poole et al. 2015). Studies examining developmental trajectories of multisensory processing for linguistic information in AS individuals have observed an improvement in the ability to integrate audio-visual syllables with age (Foxe et al. 2015; Taylor et al. 2010; although see Stevenson et al. 2014b). Cross-modal integration abilities are far from mature at birth, but rather develop over a protracted period of time and strongly depend on sensory experiences (see Stein et al. 2014, for a review). For example, experiments in animals suggested that multisensory plasticity persisted into adulthood (Stein et al. 2014). Therefore, relevant cross-modal experiences or training could compensate, at least to some extent, for an absence of typical development early in life (Stein et al. 2014). It seems therefore promising to develop longitudinal protocols intended to measure cross-modal integration abilities across the life-span in AS individuals, and eventually use those data to adapt the sensory therapies that are currently proposed in rehabilitation (Powers et al. 2012, 2016).

Multisensory atypicalities in AS individuals could, at least partly, be underpinned by alterations in temporal aspects of sensory processing. This is supported by findings demonstrating an extended temporal binding window for low-level, non-social (Kwakye et al. 2011; FossFeig et al. 2010; Greenfield et al. 2015; Noel et al. 2018; although see: Poole et al. 2018; Noel et al. 2017; Stevenson et al. 2017) and linguistic information (Woynaroski et al. 2013; Stevenson et al. 2014a; Noel et al. 2018) in AS individuals. Temporal binding windows were found to correlate with performance on the McGurk effect (Stevenson et al. 2017; Stevenson et al. 2014a), which subsequently impacted speech perception (Stevenson et al. 2017). It is not excluded that the unisensory delay observed in AS in the current study could be explain by differences between groups in temporal aspects of sensory processing. However, the paradigm used in the study does not allow to precise the origin of this general difference in RTs between the groups across modalities, and we do not exclude the possibility that it can be explained by differences in the speed of motor preparation and/or production or the use of a more cautious response criterion, rather than a sensory/perceptual deficit per se. Given that our paradigm has proved efficient to investigate withinmodal and cross-modal integration in AS individuals, an interesting avenue would be to manipulate the time interval between unisensory and multisensory pairs of stimuli and to evaluate how our results extended to different degrees of temporal synchrony between the stimuli.

\section{Within-Modal Integration in AS}

RG observed for cross-modal visuo-tactile stimuli was stronger compared to double visual and double tactile conditions in TD individuals, and no significant violation of the race model inequality was found for any redundant visual or tactile condition. These results reproduce and extend previous observations of reduced RG for within-modal compared to cross-modal information in both human (Girard et al. 2013; Forster et al. 2002; Laurienti et al. 2006) and animal populations (Gingras et al. 2009), as well as an absence of violation of Miller's race model inequality for within-modal pairs of visual (Girard et al. 2013; Murray et al. 2001; Corballis 1998; Reuter Lorenz et al. 1995; Badzakova-Traikov et al. 2005) and tactile stimuli (Girard et al. 2013; Forster et al. 2002). RG from within-modal interactions therefore seems to be explained by simple probabilistic facilitation while cross-modal integration violates such pure statistical facilitation. It is worth nothing that these results are somewhat consistent with those of studies showing that withinmodal pairs were generally associated with subadditive neural reponses, while cross-modal pairs generated additive or superadditive neural signals, at least when recorded in the superior colliculus in non-humans (Alvarado et al. 2007a, b). Moreover, the deactivation of the anterior ectosylvian sulcus (AES) and the rostral aspect of the lateral suprasylvian sulcus (rLS), which are mandatory for cross-modal integration, had no impact on the integration of within-modal pairs, suggesting fundamental differences in the circuits underlying within and cross-modal integration (Alvarado et al. 2007b). The most likely explanation seems to be that cross-modal integration combines sensory inputs from more independent sources, therefore allowing to reduce the uncertainty of the sensory estimates, while within-modal integration rather 
reflects the covariance between similar information (Ernst and Banks 2002; Alvarado et al. 2008; Girard et al. 2013; Van Atteveldt et al. 2014).

AS individuals also exhibited a RG for the within-modal conditions, which was expressed by a significant reduction in RTs in the double tactile and the double visual conditions compared to the simple tactile and visual conditions. Similar to the TD group, the RTs in the within-modal conditions did not exceed the probabilistic sum of the RTs observed in the visual or tactile conditions. However, we found an interesting difference when comparing the performance of the two groups, as cross-modal visuo-tactile stimuli produced greater RT facilitation compared to both double tactile and visual conditions in TD, while RGs for visuo-tactile and double tactile RGs were both superior to RG for the double visual condition in AS individuals. This result was not expected based on our initial assumptions. Based on previous work in TD individuals, it might be hypothesized that tactile inputs were processed more independently from one another in AS individuals, which may have led to higher RGs associated with integration of tactile stimuli (see Fig. 2). In fact, a recent study compared the performance of AS and TD participants in a discrimination task of visual and tactile stimuli that were presented concurrently with visual distractors (Poole et al. 2018). Results demonstrated that there was no difference in performance for within-modal versus cross-modal target-distractor combinations in the AS group, while the suppression of distractors was more effective when they were presented in a different modality than the target in TD individuals. However, this remains a posteriori hypothesis and it seems important to ensure that this result is replicated in subsequent studies.

When performing tasks for which spatial information is irrelevant, as is the case in the detection task used in this study, the spatial alignment of stimuli does not generally impact cross-modal integration (Forster et al. 2002; Girard et al. 2011, 2013). In contrast, regarding within-modal integration, we found in a previous study that the facilitative effect for double visual or tactile stimuli was greater when they were presented in opposite hemifields compared to the same hemifield, even if the distance between stimuli was the same for both conditions (Girard et al. 2013). Similar results were obtained in the current study. Specifically, we found no significant spatial alignment difference in RGs for the cross-modal condition, and the race model inequality was violated over the same range of RTs distributions for both aligned and misaligned visuo-tactile pairs. When looking at within-modal integration, RGs in the misaligned conditions were larger than the RGs in the aligned conditions for the double visual pairs, and although not statistically significant, this same trend was observed for double tactile pairs. No between-goup difference was found when assessing how within- and cross-modal integration were modulated by the spatial alignment of the stimuli (see SFigs 2-5). This result might seem to contrast with previous findings demonstating an increased influence of visual distractors on tactile judgments, with a difficulty for suppressing the processing of distant contralateral distractors in the AS (Poole et al. 2015). However, one major difference between the Poole et al. (2015) study and the present one is that the distance between aligned and misaligned pairs was equal in our paradigm, and the stimuli were close enough spatially so that we expect them to be integrated. We can therefore conclude that when controlling for inter-stimulus distance, the effect of spatial alignment on cross-modal processing was the same in AS and TD individuals. A possible explanation of the findings obtained in both groups is that the representations of the stimuli can strongly overlap in within-modal conditions, with both stimuli producing similar neural representation, which result in a reduce RG for the aligned conditions. However, when stimuli from the same sensory modality are presented in opposite hemifields, they are processed independently by each hemisphere, resulting in different nonoverlapping neural representations that contribute to the enhanced RG (see Girard et al. 2013 for a detailed discussion on the subject).

\section{Modality Switch Cost in AS}

Sequential stimuli presented during a simple detection task are not processed independently, as RT to a sensory input is faster when preceded by a stimulus from the same modality (Harrar et al. 2014; Otto and Mamassian 2012; Gondan et al. 2004). In contrast, when the stimuli in successive trials originate from different modalities, attention needed to shift from one modality to another leads to longer RTs (modality shift cost). Since several studies have shown impairments in the disengagement of attention in autistic population (Courchesne et al. 1994; Landry and Bryson 2004; Renner et al. 2006; Wainwright-Sharp and Bryson 1993; but see Boer-Schellekens et al. 2013; Iarocci and Burack 2004; Senju et al. 2004; Leekam et al. 2000), we investigated whether alteration in sensory integration may relate to atypical modality switch cost in AS individuals. We observed a classic modality switch cost for both visual and tactile modalities, as RTs were faster when consecutive stimuli were coming from the same modality. Importantly, this effect was similar between the two groups. This result is consistent with those from Williams et al. (2013) who demonstrated an absence of difference in the modality shift cost between AS and TD adults (although see Occelli et al. 2013 and Williams et al. 2013 for atypical modality shift cost in children with AS). These findings therefore suggest that the reduction in cross-modal integration observed in the AS is not explained by an alteration in the ability to 
switch attention from one stimulus to another (Otto and Mamassian 2012).

For both group of participants, we found that the modality switch cost was greater for tactile than for visual modality. The vast majority of studies that have investigated the modality switch cost in TD individuals are based on auditory and visual sensory modalities. However, some studies that have included the tactile modality have demonstrated larger switch cost for shifting attention away from the tactile modality in audio-tactile (Gondan et al. 2004; Spence et al. 2001) and visuo-tactile conditions (Miles et al. 2011). It was suggested that it might be particularly difficult to shift attention away from touch because it implies shifting attention from a representation of the body to the peripersonal space in which auditory and visual stimuli are encoded (Spence et al. 2001). It is also worth noting that the modality shift costs obtained in the current study remain relatively small. The use of a shorter mean inter-trial interval might have been associated with stronger effects, as it was suggested that it tends to decrease over temporal distance between two stimuli (Miles et al. 2011).

\section{Conclusion}

Our results contribute to the understanding of how AS individuals integrate information originating from different modalities. We demonstrated that AS individuals can integrate cross-modal visuo-tactile information, although this integrative process is reduced compared to TD individuals. Finding no difference in modality switch cost effect between AS and TD further reduces the possibility that atypical cross-modal integration in AS could be explained by an altered ability to switch attention from one stimulus to another. In conclusion, the present study demonstrates a selective decrease in the integration of low-level, visuotactile stimuli in AS adults.

Acknowledgments The authors would like to thank Patricia Jelenic for her help with participant recruitment and selection. OC is a research associate at the National Fund for Scientific Research-Belgium.

Author Contributions GC, SG, and OC designed research; GC, AB, MV, LM contributed in recruiting the participants; GC, MV performed the research; OC, MP, AB, LM and FL contributed unpublished reagents/analytic tools; $\mathrm{CB}$ and MP analyzed data under the supervision of OC; GC and OC wrote the first draft of the paper; all authors commented and approved the final version of the paper.

Funding This research was supported in part by the Canada Research Chair Program (FL), the Canadian Institutes of Health Research (AB, FL), the Natural Sciences and Engineering Research Council of Canada (FL, GC) and the Research Center of the University Hospital SainteJustine (OC).

\section{References}

Adolphs, R. (2002). Recognizing emotion from facial expressions: Psychological and neurological mechanisms. Behavioral and Cognitive Neuroscience Reviews, 1(1), 21-62.

Alvarado, J. C., Rowland, B. A., Stanford, T. R., \& Stein, B. E. (2008). A neural network model of multisensory integration also accounts for unisensory integration in superior colliculus. Brain Research, 1242, 13-23.

Alvarado, J. C., Stanford, T. R., Vaughan, J. W., \& Stein, B. E. (2007a). Cortex mediates multisensory but not unisensory integration in superior colliculus. Journal of Neurosciences, 27(47), 12775-12786.

Alvarado, J. C., Vaughan, J. W., Stanford, T. R., \& Stein, B. E. (2007b). Multisensory versus unisensory integration: Contrasting modes in the superior colliculus. Journal of Neurophysiology, 97(5), 3193-3205.

American Psychiatric Association. (2000). Diagnostic and statistical manual of mental disorders (4th ed.). Washington, DC: Author.

American Psychiatric Association. (2013). Diagnostic and statistical manual of mental disorders (5th ed.). Washington, DC: Author.

Badzakova-Traikov, G., Hamm, J. P., \& Waldie, K. E. (2005). The effects of redundant stimuli on visuospatial processing in developmental dyslexia. Neuropsychologia, 43, 473-478.

Bahrick, L. E. (2010). Intermodal perception and selective attention to intersensory redundancy: Implications for typical social development and autism. In G. Dans \& T. D. Wachs (Eds.), Blackwell handbook of infant development (2nd ed.). Oxford, England: Blackwell Publishing.

Bahrick, L. E., \& Todd, J. T. (2011). Multisensory processing in autism spectrum disorders: Intersensory processing disturbance as a basis for atypical development. In M. T. Dans \& M. M. Murray (Eds.), Frontiers in the neural basis of multisensory processes. London: FrancisGroup.

Baum, S. H., Stevenson, R. A., \& Wallace, M. T. (2015). Behavioral, perceptual, and neural alterations in sensory and multisensory function in autism spectrum disorder. Progress in Neurobiology, 134, 140-160.

Bebko, J. M., Weiss, J. A., Demark, J. L., \& Gomez, P. (2006). Discrimination of temporal synchrony in intermodal events by children with autism and children with developmental disabilities without autism. Journal of Child Psychology and Psychiatry, 47(1), 88-98.

Behrmann, M., Thomas, C., \& Humphreys, K. (2006). Seeing it differently: Visual processing in autism. Trends in Cognitive Sciences, 10(6), 258-264.

Boer-Schellekens, L., Keetels, M., Eussen, M., \& Vroomen, J. (2013). No evidence for impaired multisensory integration of low-level audiovisual stimuli in adolescents and young adults with autism spectrum disorders. Neuropsychologia, 51, 3004-3013.

Bonneh, Y. S., Belmonte, M. K., Pei, F., Iversen, P. E., Kenet, T., Akshoomoff, N., et al. (2008). Cross-modal extinction in a boy with severely autistic behaviour and high verbal intelligence. Cognitive Neuropsychology, 25(5), 635-652.

Botvinick, M. (2004). Neuroscience: Probing the neural basis of body ownership. Science, 305(5685), 782-783.

Brandwein, A. B., Foxe, J. J., Butler, J. S., Frey, H.-P., Bates, J. C., Shulman, L. H., et al. (2015). Neurophysiological indices of atypical auditory processing and multisensory integration are associated with symptom severity in autism. Journal of Autism and Developmental Disorders, 45, 230-244.

Brandwein, A. B., Foxe, J. J., Butler, J. S., Russo, N. N., Altschuler, T. S., Gomes, H., et al. (2013). The development of multisensory integration in high-functioning autism: High-density electrical 
mapping and psychophysical measures reveal impairments in the processing of audiovisual inputs. Cerebral Cortex, 23, 1329-1341.

Brock, J., Brown, C. C., Boucher, J., \& Rippon, G. (2002). The temporal binding deficit hypothesis of autism. Developmental Psychopathology, 14(2), 209-224.

Cascio, C. J., Foss-Feig, J. H., Burnette, C. P., Heacock, J. L., \& Cosby, A. A. (2012). The rubber hand illusion in children with autism spectrum disorders: Delayed influence of combined tactile and visual input on proprioception. Autism, 16(4), 406-419.

Cascio, C. J., Lorenzi, J., \& Baranek, G. T. (2016a). Self-reported pleasantness ratings and examiner-coded defensiveness in response to touch in children with ASD: Effects of stimulus material and bodily location. Journal of Autism and Developmental Disorder, 46(5), 1528-1537.

Cascio, C. J., Woynaroski, T., Baranek, G. T., \& Wallace, M. T. (2016b). Toward an interdisciplinary approach to understanding sensory function in autism spectrum disorder. Autism Research, 9, 920-925.

Charbonneau, G., Bertone, A., Lepore, F., Nassim, M., Lassonde, M., Mottron, L., et al. (2013a). Multi-level alterations in the multisensory processing of dynamic emotion expressions in Autism Spectrum Disorders. Neuropsychologia, 51, 1002-1010.

Charbonneau, G., Véronneau, M., Boudrias-Fournier, C., Lepore, F., \& Collignon, O. (2013b). The ventriloquist in periphery: Impact of eccentricity-related reliability on audio-visual localization. Journal of Vision, 13(12), 1-14.

Collignon, O., Charbonneau, G., Peters, F., Nassim, M., Lassonde, M., Lepore, F., et al. (2013). Reduced multisensory facilitation in persons with autism. Cortex, 49(6), 1704-1710.

Corballis, M. C. (1998). Interhemispheric neural summation in the absence of the corpus callosum. Brain, 121, 1795-1807.

Courchesne, E., Townsend, J., Akshoomoff, N. A., Saitoh, O., YeungCourchesne, R., Lincoln, A. J., et al. (1994). Impairment in shifting attention in autistic and cerebellar patients. Behavioral Neuroscience, 108, 848-865.

Dakin, S., \& Frith, U. (2005). Vagaries of visual perception in autism. Neuron, 48(3), 497-507.

Dawson, G., Webb, S. J., Carver, L., Panagiotides, H., \& McPartland, J. (2004). Young children with autism shoe atypical brain responses to fearful versus neutral facial expressions of emotion. Developmental Science, 7(3), 340-359.

De Heering, A., Dormal, G., Pelland, M., Lewis, T., Maurer, D., \& Collignon, O. (2016). A brief period of postnatal visual deprivation alters the balance between auditory and visual attention. Current Biology, 26, 3101-3105.

Dunbar, R. (2010). The social role of touch in humans and primates: Behavioral function and neurobiological mechanisms. Biobehavioral Reviews, 34(2), 260-268.

Ernst, M. O., \& Banks, M. S. (2002). Humans integrate visual and haptic information in a statistically optimal fashion. Nature, 415, 429-433.

Field, T. (2001). Touch. Cambridge, MA: MIT Press.

Forster, B., Cavina-Pratesi, C., Aglioti, S. M., \& Berlucchi, G. (2002). Redundant target effect and intersensory facilitation from visual-tactile interactions in simple reaction time. Experimental Brain Research, 143, 480-487.

Foss-Feig, J. H., Kwakye, L. D., Cascio, C. J., Burnette, C. P., Kadivar, H., Stone, W. L., et al. (2010). An extended multisensory temporal binding window in autism spectrum disorders. Experimental Brain Research, 203(2), 381-389.

Foxe, J. J., Molhom, S., Del Bene, V. A., Frey, H. P., Russo, N. N., Blanco, D., et al. (2015). Severe multisensory speech integration deficits in high-functioning school-aged children with autism spectrum disorder (ASD) and their resolution during early adolescence. Cerebral Cortex, 25(2), 298-312.
Frith, U., \& Happe, F. (1994). Autism: Beyond "theory of mind". Cognition, 50(1-3), 115-132.

Gingras, G., Rowland, B. A., \& Stein, B. E. (2009). The differing impact of multisensory and unisensory integration on behavior. The Journal of Neuroscience, 29, 4897-4902.

Girard, S., Collignon, O., \& Lepore, F. (2011). Multisensory gain within and across hemispaces in simple and choice reaction time paradigms. Experimental Brain Research, 214(1), 1-8.

Girard, S., Pelland, M., Lepore, F., \& Collignon, O. (2013). Impact of the spatial congruence of redundant targets on within-modal and cross-modal integration. Experimental Brain Research, 224(2), 275-285.

Gondan, M., Lange, K., Rösler, F., \& Röder, B. (2004). The redundant target effect is affected by modality switch costs. Psychonomic Bulletin \& Review, 11, 307-313.

Greenfield, K., Ropar, D., Smith, A. D., Carey, M., \& Newport, R. (2015). Visuo-tactile integration in autism: Atypical temporal binding may underlie greater reliance on proprioceptive information. Molecular Autism, 6, 51.

Harrar, V., Tammam, J., Pérez-Bellido, A., Pitt, A., Stein, J., \& Spence, C. (2014). Multisensory integration and attention in developmental dyslexia. Current Biology, 24, 531-535.

Hershenson, M. (1962). Reaction time as a measure of intersensory facilitation. Journal of Experimental Psychology, 63, 289.

Hertenstein, M. (2002). Touch: its communicative functions in infancy. Human Development, 45(2), 70-94.

Hertenstein, M., Keltner, D., App, B., Bulleit, B., \& Jaskolla, A. (2006). Touch communicates distinct emotions. Emotion, 6(3), 528.

Iarocci, G., \& Burack, J. A. (2004). Intact covert orienting to peripheral cues among children with autism. Journal of Autism and Developmental Disorders, 34(3), 257-264.

Iarocci, G., \& McDonald, J. (2006). Sensory integration and the perceptual experience of persons with autism. Journal of Autism and Developmental Disorders, 36(1), 77-90.

Khan, S., Michmizos, K., Tommerdahl, M., Ganesan, S., Kitzbichler, M. G., Zetino, M., et al. (2015). Somatosensory cortex functional connectivity abnormalities in autism show opposite trends, depending on direction and spatial scale. Brain, 138, 1394-1409.

Kwakye, L. D., Foss-Feig, J. H., Cascio, C. J., Stone, W. L., \& Wallace, M. T. (2011). Altered auditory and multisensory temporal processing in autism spectrum disorders. Frontiers in Integrative Neuroscience, 4(129), 1-11.

Landry, R., \& Bryson, S. E. (2004). Impaired disengagement of attention in young children with autism. Journal of Child Psychology and Psychiatry, 45, 1115-1122.

Laurienti, P. J., Burdette, J. H., Maldjian, J. A., \& Wallace, M. T. (2006). Enhanced multisensory integration in older adults. Neurobiology of Aging, 27(8), 1155-1163.

Leekam, S. R., Lopez, B., \& Moore, C. (2000). Attention and joint attention in preschool children with Autism. Developmental Psychology, 36(2), 261-273.

Lord, C., Risi, S., Lambrecht, L., Cook, E. H., Jr., Leventhal, B. L., DiLavore, P. C., et al. (2000). The autism diagnostic observation schedule-generic: A standard measure of social and communication deficits associated with the spectrum of autism. Journal of Autism and Developmental Disorders, 30(3), 205-223.

Lord, C., Rutter, M., \& Le Couteur, A. (1994). Autism diagnostic interview-revised: A revised version of a diagnostic interview for caregivers of individuals with possible pervasive developmental disorders. Journal of Autism and Developmental Disorders, 24(5), 659-685.

Magnée, M. J., de Gelder, B., van Engeland, H., \& Kemner, C. (2008). Audiovisual speech integration in pervasive developmental disorder: Evidence from event-related potentials. Journal of Child Psychology Psychiatry, 49(9), 995-1000. 
Maitre, N. L., Key, A. P., Chorna, O. D., Slaughter, J. C., Matusz, P. J., Wallace, M. T., et al. (2017). The dual nature of early-life experience on somatosensory processing in the human infant brain. Current Biology, 27(7), 1048-1054.

Marco, E. J., Hinkley, L. B., Hill, S. S., \& Nagarajan, S. S. (2011). Sensory processing in autism: A review of neurophysiological findings. Pediatric Research, 69, 48-54.

Miles, E., Brown, R., \& Poliakoff, E. (2011). Investigating the nature and time-course of the modality shift effect between vision and touch. The Quarterly Journal of Experimental Psychology, 64(5), 871-888.

Miller, J. (1982). Divided attention evidence for coactivation with redundant signals. Cognitive Psychology, 14, 247-279.

Miller, J. (1986). Timecourse of coactivation in bimodal divided attention. Perception and Psychophysics, 40(5), 331-343.

Miniussi, C., Girelli, M., \& Marzi, C. (1998). Neural site of the redundant target effect electrophysiological evidence. Journal of Cognitive Neuroscience, 10, 216-230.

Mongillo, E. A., Irwin, J. R., Whalen, D. H., Klaiman, C., Carter, A. S., \& Schultz, R. T. (2008). Audiovisual processing in children with and without autism spectrum disorders. Journal of Autism and Developmental Disorders, 38, 1349-1358.

Mottron, L., Dawson, M., Soulières, I., Hubert, B., \& Burack, J. (2006). Enhanced perceptual functioning in autism: An update, and eight principles of autistic perception. Journal of Autism and Developmental Disorders, 36(1), 27-43.

Murray, M. M., Foxe, J. J., Higgins, B. A., Javitt, D. C., \& Schroeder, C. E. (2001). Visuo-spatial neural response interactions in early cortical processing during a simple reaction time task: A high-density electrical mapping study. Neuropsychologia, 39, 828-844.

Myers, B. J. (1984). Mother-infant bonding: The status of this critical period hypothesis. Developmental Review, 4, 240-274.

Noel, J.-P., De Niear, M. A., Lazzara, N. S., \& Wallace, M. T. (2018a). Uncoupling between multisensory temporal function and nonverbal turn-taking in autism spectrum disorder. IEEE Transactions on Cognitive and Developmental Systems, 10(4), 973-982.

Noel, J. P., De Niear, M. A., Stevenson, R., Alais, D., \& Wallace, M. T. (2017). Atypical rapid audio-visual temporal recalibration in autism spectrum disorders. Autism Research, 10(1), 121-129.

Noel, J. P., Lytle, M., Cascio, C., \& Wallace, M. T. (2018b). Disrupted integration of exteroceptive and interoceptive signaling in autism spectrum disorder. Autism Research, 11(1), 194-205.

Noel, J. P., Stevenson, R. A., \& Wallace, M. T. (2018c). Atypical audiovisual temporal function in autism and schizophrenia: Similar phenotype, different cause. European Journal of Neuroscience, 47(10), 1230-1241.

O’Neill, M., \& Jones, R. S. P. (1997). Sensory-perceptual abnormalities in autism: A case for more research? Journal of Autism and Developmental Disorders, 27, 283-293.

Occelli, V., Esposito, G., Venuti, P., Arduino, G. M., \& Zampini, M. (2013). Attentional shifts between audition and vision in autism spectrum disorders. Research in Autism Spectrum Disorders, 7, 517-525.

Otto, T. U., \& Mamassian, P. (2012). Noise and correlations in parallel perceptual decision making. Current Biology, 22, 1391-1396.

Paton, B., Hohwy, J., \& Enticott, P. G. (2012). The rubber hand illusion reveals proprioceptive and sensorimotor differences in autism spectrum disorders. Journal of Autism and Developmental Disorders, 42(9), 1870-1883.

Poole, D., Gowen, E., Warren, P. A., \& Poliakoff, E. (2015). Investigating visual-tactile interactions over time and space in adults with autism. Journal of Autism and Developmental Disorder, 45, 3316-3326.
Poole, D., Gowen, E., Warren, P. A., \& Poliakoff, E. (2018). Visualtactile selective attention in autism spectrum condition: An increased influence of visual distractors. Journal of Experimental Psychology: General, 147, 1309.

Powers, A. R., Hevey, M. A., \& Wallace, M. T. (2012). Neural correlates of multisensory perceptual learning. The Journal of Neurosciences, 32(18), 6263-6274.

Powers, A. R., Hillock-Dunn, A., \& Wallace, M. T. (2016). Generalization of multisensory perceptual learning. Scientific Reports, 6, 23374.

Puts, N. A. J., Wodka, E. L., Harris, A. D., Crocetti, D., Tommerdahl, M., Mostofsky, S. H., et al. (2017). Reduced GABA and altered somatosensory function in children with autism spectrum disorder. Autism Research, 10(4), 608-619.

Raab, D. H. (1962). Statistical facilitation of simple reaction times. Transactions of the New York Academy of Sciences, 24, 574-590.

Renner, P., Klinger, L. G., \& Klinger, M. R. (2006). Exogenous and endogenous attention orienting in autism spectrum disorders. Child Neuropsychology, 12(4-5), 361-382.

Reuter Lorenz, P. A., Nozawa, G., Gazzaniga, M. S., \& Hughes, H. C. (1995). Fate of neglected targets: a chronometric analysis of rendundant target effects in the bisected brain. Journal of Experimental Psychology: Human Perception and Performance, 21, 211-230.

Rogers, S. J., Hepburn, S., \& Wehner, E. (2003). Parent reports of sensory symptoms in toddlers with autism and those with other developmental disorders. Journal of Autism and Developmental Disorders, 33, 631-642.

Schütz-Bosbach, S., Mancini, B., Aglioti, S. M., \& Haggard, P. (2006). Self and other in the human motor system. Current Biology, 16, 1830-1834.

Schütz-Bosbach, S., Musil, J. J., \& Haggard, P. (2009). Touchant-touché: The role of self-touch in the representation of body structure. Consciousness and Cognition, 18, 2-11.

Senju, A., Tojo, Y., Dairoku, H., \& Hasegawa, T. (2004). Reflexive orientating in response to eye gaze and an arrow in children with and without autism. Journal of Child Psychology and Psychiatry, 45(3), 445-458.

Shams, L., Kamitani, Y., \& Shimojo, S. (2000). What you see is what you hear. Nature, 408(6814), 788.

Simon, D. M., \& Wallace, M. T. (2016). Dysfunction of sensory oscillations in autism spectrum disorder. Neuroscience and Biobehavioral Reviews, 68, 848-861.

Spence, C., Nicholls, M. E. R., \& Driver, J. (2001). The cost of expecting events in the wrong sensory modality. Perception \& Psychophysics, 63, 330-336.

Stein, B. E., \& Meredith, M. A. (1993). The merging of the senses. Cambridge: MIT Press.

Stein, B. E., Stanford, T. R., \& Rowland, B. A. (2014). Development of multisensory integration from the perspective of the individual neuron. Nature Reviews Neuroscience, 15(8), 520-535.

Stevenson, R. A., Segers, M., Ferber, S., Barense, M. D., Camarata, S., \& Wallace, M. T. (2016). Keeping time in the brain: Autism spectrum disorder and audiovisual temporal processing. Autism Research, 9, 720-738.

Stevenson, R. A., Segers, M., Ncube, B. L., Black, K. R., Bebko, J. M., Ferber, S., et al. (2017). The cascading influence of multisensory processing on speech perception in autism. Autism, 22(5), 609-624.

Stevenson, R. A., Siemann, J. K., Schneider, B. C., Eberly, H. E., Woynaroski, T. G., Camarata, S. M., et al. (2014a). Multisensory temporal integration in autism spectrum disorders. The Journal of Neuroscience, 34(3), 691-697.

Stevenson, R. A., Siemann, J. K., Woynaroski, T. G., Schneider, B. C., Eberly, H. E., Camarata, S. M., et al. (2014b). Brief report: 
Arrested development of audiovisual speech perception in autism spectrum disorders. Journal of Autism and Developmental Disorders, 44(6), 1470-1477.

Tavassoli, T., Bellesheim, K., Tommerdahl, M., Holden, J. M., Kolevzon, A., \& Buxbaum, J. D. (2016). Altered tactile processing in children with autism spectrum disorder. Autism Research, 9(6), 616-620.

Taylor, N., Isaac, C., \& Milne, E. (2010). A comparison of the development of audiovisual integration in children with autism spectrum disorders and typically developing children. Journal of Autism and Developmental Disorder, 40(11), 1403-1411.

Thye, M. D., Bednarz, H. M., Herringshaw, A. J., Sartin, E. B., \& Kana, R. K. (2018). The impact of atypical sensory processing on social impairments in autism spectrum disorder. Developmental Cognitive Neuroscience, 29, 151-167.

Todd, J. W. (1912). Reaction time to multiple stimuli. Archives of Psychology, 3, 1-65.

Tomchek, S. D., \& Dunn, W. (2007). Sensory processing in children with and without autism: A comparative study using the short sensory profile. The American Journal of Occupational Therapy, 61, 190-200.

Townsend, J. T., \& Ashby, F. G. (1978). Methods of modeling capacity in simple processing systems. In N. J. Castellan \& F. Restle (Eds.), Cognitive theory. Hillsdale, NJ: Erlbaum.

Ulrich, R., Miller, J., \& Schröter, H. (2007). Testing the race model inequality: An algorithm and computer programs. Behavior Research Methods, 39(2), 291-302.
Van Atteveldt, N., Murray, M. M., Thut, G., \& Schroeder, C. E. (2014). Multisensory integration: Flexible use of general operations. Neuron, 81(6), 1240-1253.

Wainwright-Sharp, J. W., \& Bryson, S. E. (1993). Visual orienting deficits in high functioning people with autism. Journal of Autism and Developmental Disorders, 23, 1-13.

Wallace, M. T., \& Stevenson, R. A. (2014). The construct of the multisensory temporal binding window and its dysregulation in developmental disabilities. Neuropsychologia, 64, 105-123.

Williams, D. L., Goldstein, G., \& Minshew, N. J. (2013). The modality shift experiment in adults and children with high functioning autism. Journal of Autism and Developmental Disorders, 43, 794-806.

Woynaroski, T. G., Kwakye, L. D., Foss-Feig, J. H., Stevenson, R. A., Stone, W. L., \& Wallace, M. T. (2013). Multisensory speech perception in children with autism spectrum disorders. Journal of Autism and Developmental Disorders, 43, 2891-2902.

Publisher's Note Springer Nature remains neutral with regard to jurisdictional claims in published maps and institutional affiliations. 